\title{
Abstract
}

The situation generated by the pandemic declared in March 2020 led to a transformation of education developed in face-to-face contexts to virtual. Here is a design experience with future teachers of Preschool and Primary Education whose objective is to develop comics as a teaching resource. For this, the students are distributed in pairs and make a comic to introduce, explain or reinforce the selected mathematical content. The experimentation carried out shows as a result a variety of contexts, often relating them to other subjects such as history. Furthermore, student motivation increased compared to the usual way of working. All this, together with the ability of students to abstract when working mathematics from the comic, makes this resource a useful one to keep in mind in mathematics classrooms.

\section{Elaborando cómics en tiempo de confinamiento para aprender matemáticas en Educación Infantil y Primaria}

\section{Beatriz Sánchez-Barbero; M. José Cáceres García; J. M. Chamoso Sánchez; M. Mercedes Rodríguez Sánchez; David Rodríguez Muelas}

Universidad de Salamanca

\section{Resumen}

La situación generada por la pandemia declarada en marzo de 2020 provocó una transformación de la enseñanza desarrollada en contextos presenciales a los virtuales. Así, este trabajo muestra una experiencia desarrollada con futuros maestros de Educación Infantil y Primaria cuyo objetivo es elaborar cómics digitales como recurso didáctico para aprender matemáticas. Para ello, los alumnos se distribuyen en parejas y realizan un cómic para introducir, explicar o reforzar el contenido matemático seleccionado. La experimentación realizada muestra cómo los estudiantes eligen para el desarrollo de contenidos matemáticos una variedad de contextos reales y en muchas ocasiones los relacionan con otras materias como la historia. Además, la motivación de los alumnos aumenta comparada con la forma habitual de trabajo. Todo esto, unido a la capacidad de abstracción de los alumnos al trabajar las matemáticas desde el cómic, hace de este recurso una herramienta útil en las aulas de matemáticas.

Palabras clave: Educación Infantil y Primaria, Educación Matemática, formación docente, cómics, enseñanza virtual.

\section{MAGISTER}

Vol. 32. Núm. I: (2020). Sección extraordinaria
Key words: Preschool and Primary Education, mathematics education, teacher training, comics, virtual classroom

\section{Introducción}

Es clara la necesidad de un cambio en la enseñanza y aprendizaje de las matemáticas (NCTM, 2000), debido al desinterés de los alumnos por la materia al no encontrar una relación con el mundo real (Alsina, 2010; Morales y Villa, 2019). Si esto se traslada a aulas de formación inicial de maestros, se traduce en inquietudes sobre cómo llevar esa conexión a las aulas. Valbuena, Conde y Ortiz (2018) afirman que la calidad de la educación está relacionada con la calidad docente; una de las tareas de gran relevancia que debería asumir la formación inicial de maestros es la consideración de diferentes recursos didácticos para dotar a los futuros docentes de herramientas con las que hacer frente a situaciones que surgirán en sus aulas (Cáceres, Chamoso y Azcárate, 2010).

El cómic es una composición de viñetas, entendiéndose por estas a las imágenes encuadradas y ordenadas, que forman una historieta. En ocasiones estas viñetas van acompañadas de elementos escritos, que según la relación que guarden las viñetas puede convertirse en un relato, siendo uno de los medios más expresivos de la literatura (González y Fernández. 2018). El cómic es un recurso que, más allá del 
mero entretenimiento, puede generar conocimiento. Esto convierte al cómic en uno de los recursos educativos más motivadores pues fomenta la lectura por el apoyo visual y el interés en el contenido de la materia que trate dicha lectura, en este caso, de matemáticas (Barrero, 2002).

La utilización del cómic como recurso didáctico se considera adecuada en las aulas de matemáticas, pues la lectura matemática muestra la relación entre la materia y el entorno; acerca al alumno a las matemáticas a través de la ficción; motiva y mejora la actitud hacia las matemáticas; fomenta y desarrolla la lectura y reflexión; y facilita una enseñanza interdisciplinaria y globalizada (Muñoz, Fernández y Redondo, 2011). Además, las posibilidades y características narrativas del cómic hacen que sea una herramienta apropiada que permite trabajar contenidos curriculares concretos de todas las etapas (Bartual, 2013; Pons, 2017, citados en Sempere, Rovira-Collado y Baile, 2018).

\section{El proceso de creación de los cómics para enseñar matemáticas}

La experimentación se llevó a cabo con los alumnos de las asignaturas Matemáticas y su Didáctica del Grado en Maestro de Educación Infantil (37 alumnos) y Didáctica de las Matemáticas I y II del Grado en Maestro de Educación Primaria (108 alumnos) y del Doble Grado en Maestro de Educación Infantil y Primaria (41 alumnos), en la Escuela Universitaria de Magisterio de Zamora, perteneciente a la Universidad de Salamanca. La metodología utilizada en estas asignaturas es activa y participativa, haciendo al alumnado constructor de su propio aprendizaje. A través de la plataforma virtual Studium, basada en Moodle que utiliza la Universidad de Salamanca, (más detalle en Sánchez, Rodríguez, Cáceres, Manzanares y Chamoso, 2016), los estudiantes conocen con antelación qué se va a trabajar en cada una de las sesiones, de manera que pueden preparar los materiales necesarios para su seguimiento.

Con el objetivo de elaborar un recurso didáctico para aprender matemáticas se diseñó una tarea con la que los estudiantes debían desarrollar competencias deseables en la formación de docentes, como la competencia matemática, la competencia tecnológica y la competencia lingüística. Los estudiantes, en cada asignatura, debían organizarse en parejas y seleccionar un contenido matemático entre los trabajados en la asignatura (Tabla 1) y crear un cómic donde explicar, introducir o reforzar un contenido matemático seleccionado. Para ello había que:

- elegir escenario y protagonistas adecuados a la historia que se quiere contar.

- crear viñetas suficientes para que la historia que se cuente tenga un comienzo y un final coherente y trate el contenido matemático seleccionado de forma adecuada (entre 15 y 20 viñetas).

Tabla 1.

Contenidos matemáticos trabajados en cada asignatura implicada en el proyecto

\begin{tabular}{|c|c|c|}
\hline \multicolumn{3}{|c|}{ CONTENIDOS MATEMÁTICOS TRABAJADOS POR ASIGNATURAS } \\
\hline $\begin{array}{l}\text { Matemáticas y su Didáctica } \\
\text { del Grado en Maestro de } \\
\text { Educación Infantil }\end{array}$ & $\begin{array}{c}\text { Didáctica de las Matemáticas I del } \\
\text { Grado en Maestro de Educación } \\
\text { Primaria y } \\
\text { del Doble Grado en Maestro de } \\
\text { Educación Infantil y Primaria }\end{array}$ & $\begin{array}{c}\text { Didáctica de las Matemáticas II del } \\
\text { Grado en Maestro de Educación } \\
\text { Primaria y } \\
\text { del Doble Grado en Maestro de } \\
\text { Educación Infantil y Primaria }\end{array}$ \\
\hline 1. Números y su secuencia & 1. Números enteros & 1. Situación en el plano y en el \\
\hline 2. Comparación de números & 2. Números naturales & espacio. \\
\hline 3. Conteo & 3. Suma & 2. Posiciones relativas de rectas y \\
\hline 4. Operaciones & 4. Resta & circunferencias. \\
\hline 5. Patrones, relaciones y & 5. Multiplicación & 3. Ángulos \\
\hline funciones & 6. División exacta & 4. Sistema de coordenadas \\
\hline 6. Utilización de símbolos & 7. División no exacta & cartesianas. \\
\hline $\begin{array}{l}\text { 7. Formas de una y dos } \\
\text { dimensiones }\end{array}$ & $\begin{array}{l}\text { 8. Raíz cuadrada } \\
\text { 9. Raíz cúbica }\end{array}$ & $\begin{array}{l}\text { 5. Posiciones y movimientos. } \\
\text { 6. Formas planas y espaciales }\end{array}$ \\
\hline 8. Formas de tres dimensiones & 10. Divisibilidad & 7. Clasificación de polígonos \\
\hline $\begin{array}{l}\text { 9. Posiciones y relaciones } \\
\text { espaciales }\end{array}$ & $\begin{array}{l}\text { 11. Descomposición numérica } \\
\text { 12. Sistema decimal }\end{array}$ & $\begin{array}{l}\text { 8. Concavidad y convexidad de } \\
\text { figuras planas }\end{array}$ \\
\hline $\begin{array}{l}\text { 10. Transformaciones } \\
\text { geométricas }\end{array}$ & $\begin{array}{l}\text { 13. Números pares e impares } \\
\text { 14. Números primos }\end{array}$ & $\begin{array}{l}\text { 9. Perímetro y área. } \\
\text { 10. Cuerpos geométricos }\end{array}$ \\
\hline 11. Medida de longitud & 15. Múltiplos y divisores & 11. Cuerpos redondos \\
\hline 12. Medida de superficie & 16. Seriaciones & 12. Regularidades y simetrías \\
\hline 13. Medida de capacidad & 17. Conjuntos & 13. Medida de longitud \\
\hline 14. Medida de peso & 18. Lógica & 14. Medida de superficie \\
\hline 15. Medida del tiempo & & 15. Medida de capacidad \\
\hline 16. Organización de la & & 16. Medida de peso \\
\hline información & & 17. Medida del tiempo \\
\hline 17. Análisis d & & \\
\hline
\end{tabular}

\section{MAGISTER}

Vol. 32. Núm. I: (2020). Sección extraordinaria 
En el momento de desarrollar esta tarea, debido a la situación de pandemia con la que nos encontramos (COVID-19), las clases presenciales pasaron a ser clases virtuales, de modo que, en cada una de las asignaturas mencionadas, se realizó una sesión síncrona de dos horas de duración, en su horario ordinario, realizada a través de la herramienta de Studium, Blackboard Collaborate. En ella, se explicó la plataforma Pixton, una herramienta on-line que permite la creación de cómics de forma gratuita, y la exploración de los estudiantes de dicha plataforma pudiendo resolver posibles dificultades o dudas que se encontraran. Para la organización de las parejas y selección del contenido matemático por cada una de ellas, se creó un foro en la plataforma virtual Studium, donde las parejas explicitaron los miembros que las componían y el contenido matemático que seleccionaron. La selección se realizó por orden de intervención en el foro, de manera que el contenido trabajado por cada pareja debía ser diferente al de las demás.

Además, se dedicaron otras dos sesiones donde, a modo de tutorías, se resolvieron dudas tanto en el tratamiento de los contenidos matemáticos en los contextos reales seleccionados, como del propio uso de la herramienta de creación de cómics. Las dudas de los estudiantes que tuvieron problemas de conexión, o de otra naturaleza, fueron atendidas a través del foro, del correo electrónico de los profesores o de grupos de WhatsApp con sus compañeros.

Para valorar los trabajos se utilizaron rúbricas que consideraban la originalidad en el tratamiento del contenido, así como la profundidad en su desarrollo y la coherencia de la historieta en la que se incorporaba, la correcta utilización del lenguaje y el uso de la herramienta Pixton. Los estudiantes fueron informados de la valoración obtenida en el trabajo desarrollado mediante las herramientas de evaluación disponibles en la plataforma virtual Studium y fueron atendidos mediante tutorías individuales para dar respuesta a las posibles preguntas.

\section{Resultados}

Los estudiantes, en cada materia, realizaron cómics con la herramienta Pixton de acuerdo con lo solicitado, si bien la calidad de estos no fue homogénea. Los cómics mostraron la capacidad y originalidad de los futuros maestros, así como la motivación e interés en la creación de estos. Las historietas pusieron de manifiesto potencialidades y dificultades de los estudiantes tanto en el desarrollo de contenidos matemáticos como en la comunicación lingüística. Los cómics se situaron en diversos contextos, elegidos para trabajar los distintos contenidos matemáticos vinculados a otras materias, por ejemplo, mediante viajes a Egipto trabajaron los cuerpos geométricos o la superficie de sombra de una pirámide; contextualizada en la cocina trabajaron las reglas de tres o a reducción a la unidad relacionándolo con la nutrición; protagonizadas por dinosaurios, y relacionándola con la prehistoria, trabajaron contenidos como cantidades o el sistema métrico decimal, entre otras. También permitieron detectar errores de comprensión en determinados contenidos, como un mal uso de las unidades de medida al calcular la superficie del cristal roto de una ventana, la unidad que marcaron fueron metros en lugar de metros al cuadrado; confusiones con el sistema métrico decimal igualando kilogramos con litros de forma general; expresiones del sistema de numeración romano que van en contra de las normas del mismo; pero también, comprobar la adquisión de otros, asî como la capacidad para trabajarlos con niños de educación infantil o primaria.

Un ejemplo de esto último es el cómic de "Adri y los números pares e impares". La historieta contextualiza al protagonista y a sus padres en un viaje en coche hacia la casa de su abuela; Adri, por las prisas, en lugar de un par de calcetines del mismo color, se los puso de colores diferentes y le surge la duda de por qué se dice par de calcetines... es ahí donde su padre comienza a contar la historia de "Las familias pares e impares" (Figura 1).

Los estudiantes se mostraron muy motivados y al finalizar la entrega de los trabajos, solicitaron una puesta en común para poder disponer de cómics que trataran contenidos diferentes al propio y poder utilizarlos como recursos didácticos para las aulas de matemáticas en su futuro como docentes. Para ello, en cada asignatura se creó una carpeta con todos los cómics y se puso a disposición de los estudiantes en la plataforma digital Studium.

\section{MAGISTER}

Vol. 32. Núm. I: (2020). Sección extraordinaria 

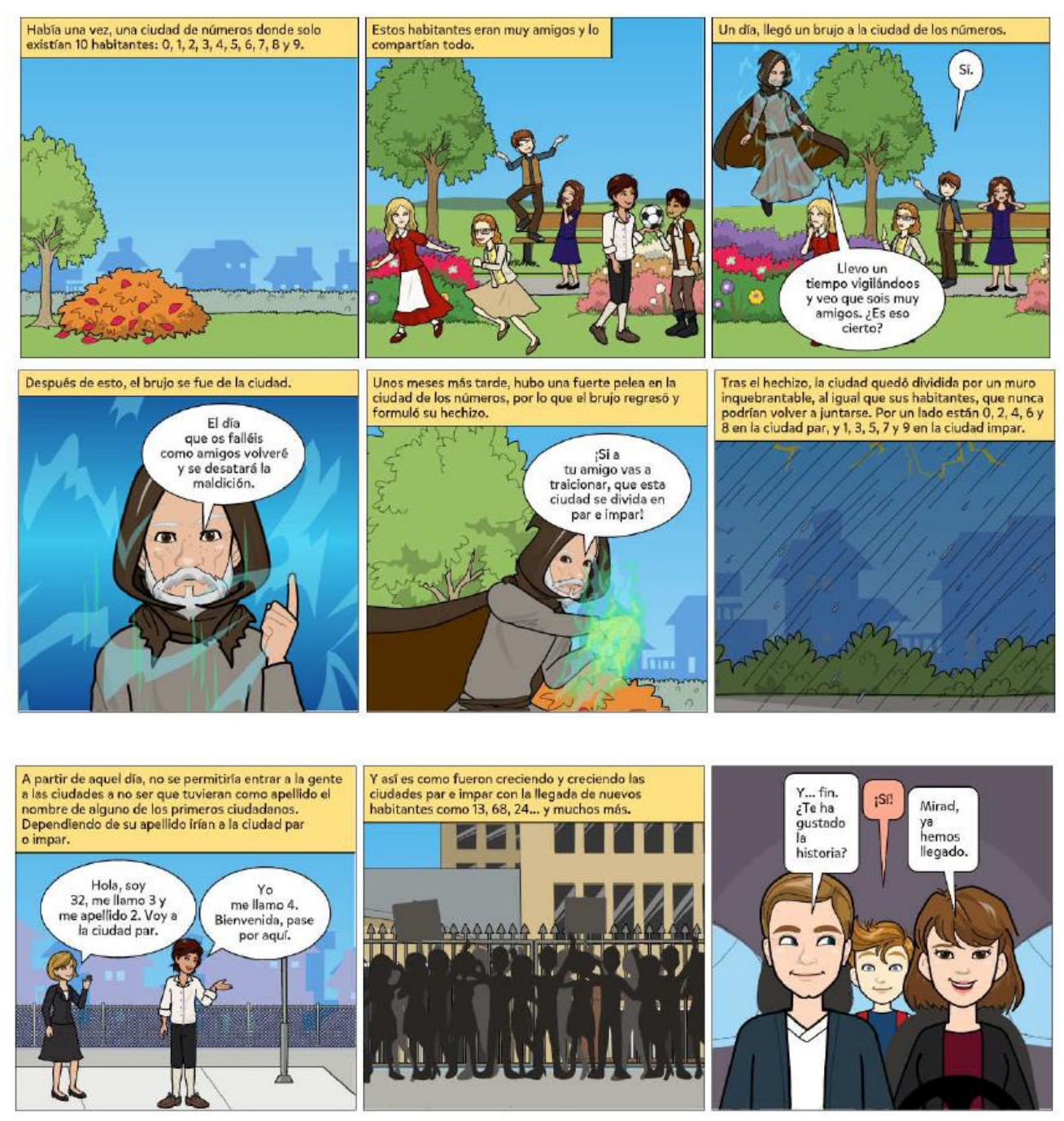

Figura 1. Cómic “Adri y los números pares e impares”

\section{Conclusiones}

El objetivo del proyecto era elaborar cómics como recurso didáctico para explicar, introducir o reforzar un contenido matemático en aulas de Educación Infantil o Primaria. La forma de trabajo en la elaboración de un material didáctico permitió una participación significativa y colaborativa (Sánchez, Chamoso, Cáceres y Rodríguez, 2015). Fue llamativo ver el interés y la motivación de los futuros maestros por la elaboración de cómics como recursos didácticos para el proceso de enseñanza-aprendizaje de las matemáticas, algo que distaba de su comportamiento cuando se les solicitaba trabajar con metodologías tradicionales. Asimismo, se considera la utilidad de este recurso para generar conocimiento, para fomentar la capacidad de abstracción siendo capaces de relacionar imagen y significado, entender diferentes modelos narrativos y su multidisciplinaridad, entre otras.

Sería conveniente experimentar los cómics creados por los estudiantes con niños de educación infantil y primaria, algo que fue imposible por la situación generada por la COVID-19, pero que se espera poder hacer en el futuro con la intención de comprobar si este recurso educativo consigue motivación e interés y un aprendizaje significativo, en cuyo caso, su introducción en las aulas de matemáticas podría ser uno de los posibles cambios que la educación necesita.

La experimentación no se pudo realizar forma presencial, como estaba previsto, sin embargo, se desarrolló de forma adecuada con las herramientas virtuales. Esta tarea se mantendrá en el futuro dentro de todas las asignaturas de los Grados en Maestro en Educación Infantil y Primaria, ya sea con enseñanza presencial o virtual, para asî poder generar una base literaria elaborada y orientada a la enseñanza de diferentes contenidos matemáticos.

\section{Referencias}

Alsina, A. (2010). La pirámide de la Educación Matemática. Una herramienta para ayudar a desarrollar la competencia matemática. Aula de Innovación Educativa, $189,12-16$.

Bartual R. (2013). Narraciones gráficas. Del códice medieval al cómic. Madrid: Factor crítico.

\section{MAGISTER}

Vol. 32. Núm. I: (2020). Sección extraordinaria 
Cáceres, M.J., Chamoso, J.M. y Azcárate, P. (2010): Analysis of the revisions that pre-service teachers of Mathematics make of their own project included in their learning portfolio. Teaching and Teacher Education 26(5), 1186-1195.

González, J.L. y Fernández de Simón, F. (2018). Relación entre cómic y enunciado matemático. Estudio y caso práctico. Revista Digital Matemática e Internet $18(1), 1-15$

Morales, R. y Villa, C. (2019). Juegos de rol para la enseñanza de las matemáticas. Education in the Knowledge Society, 20, 1-13.

Muñoz, J., Fernández, A., y Redondo, A. (2011). Leer en matemáticas. Revista Clave 21. Reflexiones y experiencias en educación, 1-33.

N.C.T.M. (2000). Principles and Standards for School Mathematics. Virginia: Reston, NCTM.

Pons, Á. (2017). La cárcel de papel. Diario de un lector de tebeos (2002-2016). Madrid: Confluencias.

Sánchez, B., Rodríguez M.M., Cáceres, M.J., Manzanares, J. y Chamoso, J.M. (2016). Una plataforma virtual con soporte Moodle para mejorar la formación de maestros de matemáticas. Comunicación presentada en el XIII Congreso Regional de Matemáticas, Ávila, España.

Valbuena, S., Conde, R. y Ortiz, J. (2018). La Investigación en educación matemática y Práctica Pedagógica, perspectiva de licenciados en Matemáticas en formación. Revista Educación y Humanismo, 20(34), 201-215.

\section{Agradecimientos}

Los autores son miembros del Grupo de Investigación Reconocido de Matemática Educativa de la Universidad de Salamanca (GIRME) y participan en los proyectos: Erasmus + "New Rules for Assessing Mathematical Competencies (RULESMATH)" (2017-1-ESO1-KA203-038491), y Junta de Castilla-León "Análisis de tareas matemáticas planteadas por estudiantes para maestro a partir de procesos reflexivos" (Q3718001E).

*Autor de contacto: Beatriz Sánchez-Barbero, beatrizsanchezb@usal.es

\section{MAGISTER}

Vol. 32. Núm. I: (2020). Sección extraordinaria 